\title{
Finding orbital motion of sub-stellar companions - the case of TWA 5B
}

\author{
T. Schmidt, R. Neuhäuser and M. Mugrauer \\ Astrophysikalisches Institut und Universitäts-Sternwarte, Universität Jena, \\ Schillergäßchen 2-3, 07745 Jena, Germany \\ email: tobi@astro.uni-jena.de
}

\begin{abstract}
TWA 5B is a brown dwarf companion of $\mathrm{H}=12 \mathrm{mag}, 2$ " off the $\sim 5$ mag brighter triple star CoD-33 7795 (=TWA 5), a member of the TW Hydrae association of T Tauri stars at $\sim 55$ pc. This object is the first brown dwarf around a pre-main-sequence star (confirmed by common proper motion) ever found. In the last year we have newly reduced VLT NaCo data originally taken in 2003 and combined it with all the available astrometric data of the system to investigate possibly detectable orbital motion of the system. Indeed we were able to find linear orbital motion of the system combining data from HST, VLT and Gemini-North.
\end{abstract}

Keywords. stars: low-mass, brown dwarfs, stars: pre-main-sequence, stars: imaging, binaries: close, stars: individual (TWA 5A, TWA 5B)

\section{Introduction}

In 1999, at the time when only two brown dwarfs were confirmed to be companions to normal stars by both spectroscopy and proper motion [Gl 229B (Nakajima et al. 1995), G 196-3 B (Rebolo et al. 1998)], Lowrance et al. (1999) (here L99) and Webb et al. (1999) (W99) suggested independently a sub-stellar companion of TWA 5 in the $\sim 8-10 \mathrm{Myr}$ young TW Hydrae association (Fig. 1). The companion TWA $5 \mathrm{~B}$ is $\sim 5$ mag fainter than the primary star in the infrared, and its IHJK colors are consistent with spectral type M8 to M8.5 (L99, W99). Weintraub et al. (2000) presented additional HST NICMOS narrow-band filter photometry, also consistent with a young late M-type brown dwarf. Neuhäuser et al. (2000) presented for the first time infrared spectra and proper motion of the $\mathrm{H}=12$ mag object 2 " off the brighter spectroscopic binary star TWA 5 finding the object to be co-moving with TWA 5A from observations with FORS and ISAAC and hence to be the 4th brown dwarf companion around a normal star confirmed by spectrum and proper motion. They derived the mass of TWA 5B to be between $\sim 15$ and $40 \mathrm{M}_{J u p}$ assuming a distance of $55 \pm 16 \mathrm{pc}$ estimated from the observation of four other members of the TW Hydrae association by Hipparcos and taking into account the age of TWA. After TWA 5A was resolved as binary (Macintosh et al. 2001) with $~ 55$ mas separation, recently Torres et al. (2003) and Mohanty et al. (2003) reported that TWA 5A is a triple, with one of the resolved stars being a spectroscopic binary. Here, we present first evidence for orbital motion of the young brown dwarf companion TWA 5B.

\section{Methods and instruments}

We gathered all the available archival observation results from Lowrance et al. (1999), Weintraub et al. (2000), Lowrance et al. (2001), Macintosh et al. (2001), Neuhäuser et al. (2000), Brandeker et al. (2003) and Neuhäuser et al. (2001). From these publications we took separation and position angle (measured from north over east to south) data and 
combined it with a new data point from data taken at VLT-UT4 with NaCo by Masciadri et al. (2005) and rereduced by us to look for first indications of orbital motion of the brown dwarf TWA 5B around its primary.
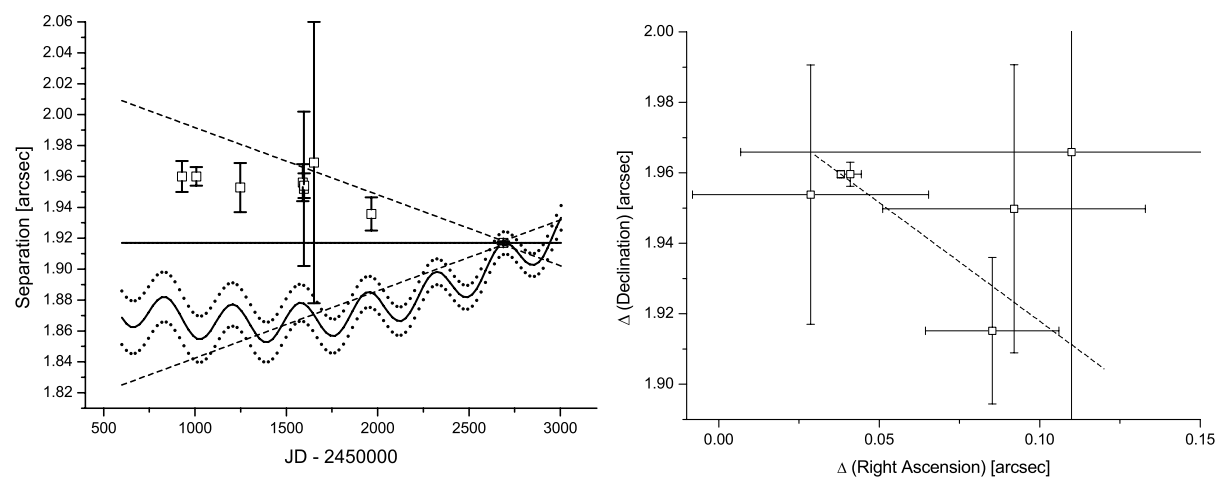

Figure 1. Left: Separations from the literature and our reduction of the 2003 data. The constant straight line indicates the case of bound motion (common proper motion). The wavy continuous line is the change expected if the sub-stellar B component is a non-moving background star. The opening cone enclosed by the dotted lines are its estimated errors. The waves of this cone show the differential parallactic motion which has to be taken into account if one of the components is a non-moving background star. The opening short dashed cone indicates the amplitude of possible orbital motion in the case of circular edge-on orbit. Right: Position of TWA 5B relative to TWA 5A.

\section{Conclusions}

From separation measurements the background hypothesis can be rejected by $6 \sigma$ and from position angle by $25 \sigma$. The deviations from the co-moving case of constant separation and position angle give more than $7 \sigma \& 10 \sigma$ significance respectively for the presence of orbital motion. A linear fit to the nine separation measurements found give a reduced $\chi^{2}$ of 0.2833 for a declining separation of 9.71 mas/yr corresponding to a probability of $96.1 \%$. In the near future curvature may be observable in the orbital motion of TWA 5B as final proof of companionship.

\section{References}

Brandeker, A.; Jayawardhana, R.; Najita, J. 2003, AJ, 126, 2009

Lowrance, P. J.; McCarthy, C.; Becklin, E. E. et al. 1999, ApJ, 512, 69 (L99)

Lowrance, P.; Becklin, E. E.; Schneider, G. et al. 2001, ASPC, 244, 289

Macintosh, B.; Max, C.; Zuckerman, B. et al. 2001, ASPC, 244, 309

Masciadri, E.; Mundt, R.; Henning, T. et al. 2005, ApJ, 625, 1004

Mohanty, S.; Jayawardhana, R.; Barrado y Navascus, D. 2003, ApJ, 593, 109

Nakajima, T.; Oppenheimer, B. R.; Kulkarni, S. R. et al. 1995, Nature, 378, 463

Neuhäuser, R.; Guenther, E. W.; Petr, M. G. et al. 2000, A\&A, 360, 39

Neuhäuser, R.; Potter, D.; Brandner, W. 2001, astro.ph, (010)6304

Rebolo, R.; Zapatero Osorio, M. R.; Madruga, S. et al. 1998, Science, 282, 1309

Torres, G.; Guenther, E. W.; Marschall, L. A. et al. 2003, AJ, 125, 825

Webb, R. A.; Zuckerman, B.; Platais, I. et al. 1999, ApJ, 512, 63 (W99)

Weintraub, D. A.; Saumon, D.; Kastner, J. H.; Forveille, T. 2000, ApJ, 530, 867 